\title{
Netilmicin/dexamethasone fixed combination in the treatment of conjunctival inflammation
}

This article was published in the following Dove Press journal:

Clinical Ophthalmology

22 June 2013

Number of times this article has been viewed

\section{Francesco Faraldi \\ Vincenzo Papa ${ }^{2}$ \\ Daria Rasà ${ }^{2}$ \\ Debora Santoro² \\ Simona Russo ${ }^{2}$ \\ 'Struttura Complessa Oculistica \\ III, Presidio Ospedaliero Oftalmico, Torino, Italy; ${ }^{2}$ Medical Affairs, SIFI SpA, Aci S Antonio, Catania, Italy}

Correspondence: Vincenzo Papa Medical Affairs - SIFI SpA, Via Ercole Patti 36, 95020 Lavinaio, Catania, Italy Tel +390957922375

Fax +39095 789345 I

Email vincenzo.papa@sifigroup.com
Purpose: To compare the efficacy and safety of $0.1 \%$ dexamethasone $/ 0.3 \%$ netilmicin (Netildex), with that of $0.1 \%$ dexamethasone $/ 0.3 \%$ tobramycin (Tobradex) in the treatment of external ocular inflammation requiring antibiotic therapy.

Methods: In this randomized, double-blind study, 139 subjects with conjunctival inflammation associated with signs of ocular infection were treated with Netildex $(n=71)$ or Tobradex $(n=68)$ four times daily for 6 days. The primary efficacy analysis was based on the percentage of patients with at least a $50 \%$ decrease in conjunctival hyperemia at the endpoint visit (Day $6[ \pm 1]$ ) with respect to baseline (responder rate). An equivalence margin of $20 \%$ was set for this study. A follow-up visit was performed at Day $14( \pm 1)$. Other efficacy parameters were: conjunctival edema, conjunctival discharge, lid hyperemia, lid edema, presence of ocular infection, and symptoms of ocular discomfort. Safety evaluations included intraocular pressure, visual acuity, and adverse events.

Results: At Day 6, a decrease of conjunctival hyperemia was observed in $87.3 \%$ and $90.9 \%$ of the patients treated with Netildex and Tobradex, respectively. The $95 \%$ confidence interval for the difference between groups $(-15.3 \div 8.0)$ satisfied the equivalence hypothesis. Subjects treated with Netildex had a better control of lid hyperemia $(P=0.016)$, tearing $(P=0.001)$, burning $(P=0.007)$, and stinging $(P=0.004)$. No adverse reactions were observed during the study except one case of keratitis in the Tobradex group.

Conclusion: Netildex was as effective and safe as Tobradex in reducing signs and symptoms in patients with conjunctival inflammation when ocular infection was suspected.

Keywords: conjunctivitis, dexamethasone, netilmicin, red eye

\section{Introduction}

Red eye is one of the most common and nonspecific ocular conditions encountered in emergency and outpatient settings, and it may be related to several different pathological conditions. ${ }^{1-4}$ Conjunctivitis is the most common cause of red eye; this condition is usually benign, and can be managed by primary care physicians. ${ }^{1-5}$ In the majority of cases, conjunctivitis is of viral origin. A bacterial etiology is normally suggested by the presence of purulent discharge, but the nature of discharge is not clinically useful in determining the cause. ${ }^{1,3}$ Despite the fact that conjunctivitis is self-limiting, a topical broad-spectrum antibiotic is usually prescribed. ${ }^{5}$ In addition, since active conjunctivitis is better controlled with preparations containing steroids, topical steroidal/antibiotic combinations are often used. ${ }^{6}$ Such combinations have several advantages over the use of single components, including better compliance, lower costs, and reduction of the potential wash-out effect. ${ }^{6,7}$ The choice among the different steroidal/antibiotic fixed 
combinations available depends on the bacterial resistance pattern to the antibiotic included in the formulation. ${ }^{4}$ A relatively new steroid/antibiotic fixed combination containing dexamethasone and netilmicin has been available in some European Union (EU) countries since 2006 (Netildex, Società Industria Farmaceutica Italiana [SIFI] SpA, Catania, Italy), and is appropriate for all inflammatory ocular conditions for which a corticosteroid is indicated and where superficial bacterial ocular infection or a risk of bacterial ocular infection exists. The antibiotic component of such combination is netilmicin, a third-generation aminoglycoside characterized by a wide spectrum of activity, including methicillin-resistant strains. ${ }^{8-11}$ This product is effective in controlling ocular inflammation after cataract surgery. ${ }^{12,13}$ The aim of the present multicenter, double-blinded equivalence study was to compare the efficacy of dexamethasone/netilmicin with that of Tobradex in the treatment of external ocular inflammation requiring antibiotic treatment.

\section{Materials and methods Study design}

The study was a multicenter, randomized, double-blind equivalence trial. Six clinical sites in Italy and one clinical site in Rumania were involved. The study protocol was approved by the Ethical Committee of the Clinical Trial Commission of Regione Piemonte (Italy, protocol no 5540/28.3) and conducted according to the Declaration of Helsinki and Good Clinical Practice. Written informed consent was obtained from all patients. The study included three visits: Day 1, Day 6 (endpoint visit), and Day 14 (follow-up visit).

\section{Patients and treatments}

A total of 139 patients with signs of conjunctival inflammation with discharge and chemosis requiring an antibiotic treatment were enrolled in the study. The demographic characteristics of these patients are shown in Table 1. Exclusion criteria for entering in the study were intraocular pressure (IOP) $>24 \mathrm{mmHg}$, known or suspected allergy to benzalkonium chloride or aminoglycosides, use of systemic decongestants, and use of anti-inflammatory agents or any ophthalmic medications (other than ocular lubricants) within 7 days prior to study entry. Eligible patients were randomly assigned to receive either Netildex, containing $0.1 \%$ dexamethasone plus $0.3 \%$ netilmicin $(n=71)$ or Tobradex (Alcon Laboratories, Inc, Fort Worth, TX, USA) containing $0.1 \%$ dexamethasone plus $0.3 \%$ tobramycin $(n=68)$. Both products were preserved with benzalkonium chloride $(0.005 \%$ in Netildex and $0.01 \%$ in Tobradex) and were packaged in an
Table I Demographic data (randomized patients)

\begin{tabular}{llll}
\hline & $\begin{array}{l}\text { Netildex } \\
(\mathbf{n}=\mathbf{7} \mathbf{)})\end{array}$ & $\begin{array}{l}\text { Tobradex } \\
(\mathbf{n}=\mathbf{6 8})\end{array}$ & $\begin{array}{l}\text { All Patients } \\
(\mathbf{n}=139)\end{array}$ \\
\hline Sex & & & \\
Female & 33 & 34 & 67 \\
Male & 38 & 34 & 72 \\
Age (years) & & & \\
Mean & 52.4 & 49.4 & 50.9 \\
SD & 15.4 & 17.6 & 16.5 \\
Range & $20-81$ & $18-82$ & $18-82$ \\
Race & & & 137 \\
Caucasian & 69 & 68 & 2 \\
$\quad$ Other & 2 & 0 & \\
\hline
\end{tabular}

Abbreviation: SD, standard deviation.

identical fashion to guarantee an appropriate masking for both patients and investigators. In case of bilateral involvement, both eyes were treated with the assigned treatment and the eye with the higher score of conjunctival hyperemia was chosen for statistical evaluation. In the presence of equal scores, the right eye was evaluated.

The presence of ocular infection was assessed by performing a conjunctival swab (Culture Swab ${ }^{\circledR}$ Becton Dickinson, Franklin Lakes, NJ, USA) at Day 1 for all patients; in patients with a positive conjunctival swab at baseline, another swab was taken at the follow-up visit (Day $14[ \pm 1]$ ). The identification of microorganisms grown in culture was performed using the analytical profile index system. Multiple semiautomated systems (Vitek ${ }^{\circledR}$, Biomerieux, Durham, NC, USA) or Kirby-Bauer disk diffusion method were adopted to perform antimicrobial susceptibility tests. An ocular specimen was considered culture positive if the colony forming units count reached the threshold values described by Cagle and Abshire. ${ }^{14}$

The treatment with the two steroid/antibiotic combinations started immediately after the swab collection and continued for $6( \pm 1)$ days four times daily. The therapy to be adopted during the follow-up period (Day 6 to 14) was established according to the swab culture results. Patients with positive cultures continued the assigned treatment to Day 14; the steroid/antibiotic combination was discontinued in patients with negative cultures.

\section{Efficacy and safety variables}

All patients were individually assessed by the same physician. Conjunctival hyperemia was chosen as the primary efficacy parameter of the study and was evaluated after $6( \pm 1)$ days of treatment (endpoint evaluation) and at the follow-up visit at Day $14( \pm 1)$ by using a categorical semiquantitative grading scale: $0=$ absence of inflammation; 
$1=$ mild inflammation (some vessel injected); 2 = moderate inflammation (diffuse injection of vessels, but individual vessels are still discernible); $3=$ severe inflammation (intense injection of vessels, individual vessels not easily still discernible). Conjunctival edema, conjunctival discharge, lid hyperemia, lid edema, and ocular infection, as well as symptoms of ocular discomfort (pain, photophobia, tearing, burning, stinging, and foreign body sensation), were adopted as secondary efficacy parameters, and were graded using a categorical semiquantitative scale. The overall safety and ocular tolerance were assessed by monitoring intraocular pressure (IOP), visual acuity, and adverse events. IOP was measured by using a calibrated Goldmann tonometer (mean of two measurements). An IOP elevation $>6 \mathrm{mmHg}$ was considered clinically significant.

\section{Statistics}

All statistical analyses were performed using SAS Business Analytics software (version 9.1; SAS Institute Inc, Cary, NC, USA). The sample size calculation was based on the primary efficacy parameter (ie, decrease in conjunctival hyperemia score $\geq 50 \%$ ) considering a two-sided significance level of $5 \%$, a power of $90 \%$, and an equivalence margin of $20 \%$. Assuming that $90 \%$ of patients treated with Tobradex would present a decrease in conjunctival hyperemia score $\geq 50 \%$ at Day $6( \pm 1)$ and no difference with respect to the test treatment, a sample size of 98 evaluable patients (49 per group) was required.

Patients with valid measurements at visit two (Day $6[ \pm 1]$ ) adhering to all protocol conditions were included in the per-protocol (PP) population, whereas patients with only minor or mild deviations (as fair compliance) were included in the full-analysis (FA) population. Primary analysis was performed on both populations. Secondary efficacy evaluations were conducted only in the FA population. Safety and local tolerance were assessed in the safety population (patients receiving at least one dose of the study drug). The primary efficacy variable was the responder rate, defined as the percentage of patients with at least a $50 \%$ decrease in conjunctival hyperemia at Day $6( \pm 1)$ (endpoint visit) with respect to baseline. A chi-square test was used to compare the responder rates of the two treatments, and a $95 \%$ confidence interval (CI) was used for the difference provided to assess the equivalence. In addition, a Wilcoxon rank-sum test and analysis of variance were used to compare treatments with respect to the score differences and the score percent variation from baseline in conjunctival hyperemia, respectively. The Pratt-Wilcoxon test was performed to compare conjunctival hyperemia scores within each treatment group (baseline versus Day $6[ \pm 1])$. Wilcoxon rank-sum test and Pratt-Wilcoxon test were performed, respectively, for between and within group analysis of further efficacy parameters and global subjective tolerance. The incidence of ocular infection was compared between treatments using the Fisher's Exact test. Differences between treatments for the IOP were evaluated by means of analysis of covariance, including treatment as a fixed effect and the IOP assessed at Day 1 as covariate. Withingroup analysis of IOP was performed using the paired $t$-test to detect significant differences with respect to baseline. Overall, the level of statistical significance $\alpha$ was set at $5 \%$.

\section{Results}

Thirty-four of the 139 (24.4\%) randomized patients were excluded from the efficacy evaluation due to major protocol violations (missed visit at day 6). The FA and PP datasets consisted of 110 patients (55 in the Netildex group and 55 in the Tobradex group) and 105 patients (51 in the Netildex group and 54 in the Tobradex group), respectively.

The primary efficacy analysis was based on the percentage of patients with at least a $50 \%$ decrease in conjunctival hyperemia at the endpoint visit (Day $6[ \pm 1]$ ) with respect to baseline (responder rate). Accordingly, the two products produced a comparable reduction of the conjunctival hyperemia score $(>70 \%$ ); within each group of treatment, this effect was highly statistically significant $(P<0.001)$ (Table 2 and Figure 1). A further decrease of the score was observed at the follow-up visit (Day 14), suggesting that there was no rebound effect after cessation of therapy (Figure 1). The responder rate (FA dataset) was equal to $87.3 \%$ in the Netildex group and to $90.9 \%$ in the Tobradex group with

Table 2 Effect on conjunctival hyperemia

\begin{tabular}{|c|c|c|c|c|}
\hline \multirow{2}{*}{$\begin{array}{l}\text { Grading scale } \\
\text { (\% variation) }\end{array}$} & \multirow{2}{*}{$\frac{\text { Netildex }}{\text { Mean } \pm \text { SD }}$} & \multirow{2}{*}{$\frac{\text { Tobradex }}{\text { Mean } \pm \text { SD }}$} & \multirow[t]{2}{*}{$P$-value* } & \\
\hline & & & & \\
\hline FA population & $-71.8 \pm 31.1$ & $70.0 \pm 27.7$ & 0.566 & \\
\hline PP population & $-71.6 \pm 31.5$ & $70.4 \pm 27.8$ & 0.632 & \\
\hline $\begin{array}{l}\text { Responder } \\
\text { rate }\end{array}$ & $\%(n / N)$ & $\%(n / N)$ & $P$-value ${ }^{\dagger}$ & $\begin{array}{l}\text { Difference } \\
95 \% \mathrm{Cl}\end{array}$ \\
\hline FA dataset & $87.3(48 / 55)$ & $90.9(50 / 55)$ & 0.541 & $-3.6(-15.3 \div 8.0)$ \\
\hline PP dataset & $86.3(44 / 5 I)$ & $90.7(49 / 54)$ & 0.472 & $-4.5(-16.7 \div 7.7)$ \\
\hline
\end{tabular}

Notes: Conjunctival hyperemia was evaluated by using a categorical semiquantitative grading scale as described in Materials and methods. Grading scale (\% variation) indicates the percentage of variation for conjunctival hyperemia grading scale at Day 6 from baseline. Responder rate indicates the percentage of patients with an improvement $\geq 50 \%$ of conjunctival hyperemia grading scale at Day 6 over baseline. *Analysis of variance; ${ }^{\dagger}$ Chi-square test.

Abbreviations: $\mathrm{Cl}$, confidence interval; $\mathrm{FA}$, full analysis; $\mathrm{n} / \mathrm{N}$, number over total; PP, per protocol; SD, standard deviation. 


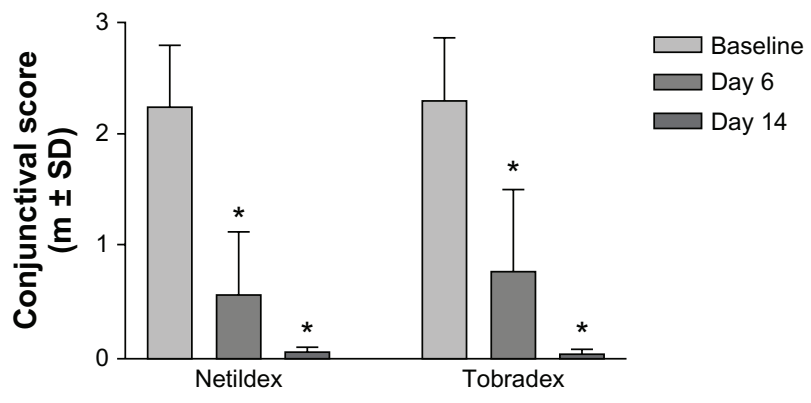

Figure I Effect of steroid/antibiotic combinations on conjunctival score. Notes: Conjunctival hyperemia was evaluated by using a categorical semiquantitative grading scale ( 0 = absent; I = mild; 2 = moderate; 3 = severe) at baseline, after 6 days of treatment with Netildex or Tobradex, and after 8 more days (follow-up visit at Day 14). Data are expressed as mean score \pm SD. *Pratt-Wilcoxon test: $P<0.000$ I (vs baseline).

Abbreviation: SD, standard deviation.

a difference of $-3.6(95 \% \mathrm{CI},-15.3 \div 8.0)$ satisfying the equivalence hypothesis (Table 2). Similar results were observed also in the PP population (Table 2).

Positive cultures at Day 1 were identified in approximately one-third of patients; the most common bacteria isolated were Staphylococcus epidermidis (eleven cases) and Staphylococcus aureus (nine cases). The classification of a swab as culture positive had little influence on the rate of clinical responsiveness. For both treatment groups, the responder rate obtained in the subgroup of patients classified as culture negative was higher than the rate observed in the subgroup of patients classified as culture positive (Table 3); however, such differences were not statistically significant. In the majority of patients with positive culture, both treatments produced a complete eradication of infections (eradication rate $91.6 \%$ and $87.5 \%$ with Netildex and Tobradex, respectively).

Table 3 Effect of the presence of ocular infection on the responder rate (FA dataset)

\begin{tabular}{|c|c|c|c|c|}
\hline & \multirow{2}{*}{$\begin{array}{l}\text { Netildex } \\
\text { Responder } \\
\text { rate }(\mathrm{n} / \mathrm{N})\end{array}$} & \multirow{2}{*}{$\begin{array}{l}\text { Tobradex } \\
\text { Responder } \\
\text { rate }(n / N)\end{array}$} & \multirow[t]{2}{*}{$P$ value* } & \multirow{2}{*}{$\begin{array}{l}\text { Difference } \\
95 \% \mathrm{Cl}\end{array}$} \\
\hline & & & & \\
\hline $\begin{array}{l}\text { Culture- } \\
\text { positive }\end{array}$ & $81.2(13 / 16)$ & $82.3(14 / 17)$ & 0.935 & I.I $(25.2 \div 27.4)$ \\
\hline $\begin{array}{l}\text { Culture- } \\
\text { negative }\end{array}$ & $89.7(35 / 39)$ & $94.7(36 / 38)$ & 0.395 & $5.3(-6.8 \div 17.3)$ \\
\hline
\end{tabular}

Notes: Conjunctival hyperemia was evaluated by using a categorical semiquantitative grading scale as described in Materials and methods. The responder rate indicates the percentage of patients with an improvement $\geq 50 \%$ of conjunctival hyperemia grading scale over baseline. The presence of ocular infection was assessed by performing a conjunctival swab at Day I. The identification of microorganisms grown in culture was performed using the API system. An ocular specimen was considered culture positive if the CFU count reached the threshold values described by Cagle. ${ }^{13} *$ Chi-square test. Abbreviations: API, analytical profile index; CFU, colony forming unit; $\mathrm{Cl}$, confidence interval; FA, full-analysis dataset; $n / N$, number over total.
Further efficacy evaluations were conducted only in the FA dataset. Comparable results between the two groups were observed for all the variables examined (data not shown). However, in the within analysis (change from baseline at Day 6), patients treated with Netildex received lower scores than patients treated with Tobradex for lid hyperemia ( $P=0.016)$, tearing $(P=0.001)$, burning $(P=0.007)$, and stinging $(P=0.004)$ (Table 4$)$.

Safety was assessed in all 139 randomized patients. Only one patient (treated with Tobradex) experienced keratitis as adverse event.

Pre-surgery IOP values in patients treated with Netildex and Tobradex were $14.9( \pm 1.8) \mathrm{mmHg}$ and $14.4( \pm 2.0)$ $\mathrm{mmHg}$ (mean \pm standard deviation), respectively. At the end of the study, IOP values were $14.7( \pm 2.0) \mathrm{mmHg}$ in the Netildex group and $14.3( \pm 1.9) \mathrm{mmHg}$ in the Tobradex group.

\section{Discussion}

External ocular inflammation is common in adults and children and may have a variety of etiologies, including bacterial infection. ${ }^{5}$ Effective treatment requires suppression of ocular surface inflammation and, if present, bacterial eradication. Microbiological evaluation is not routinely performed; therefore, a combination of steroid and antibiotic treatment is preferred in most cases. ${ }^{3}$ The practical advantages of the administration of such fixed combinations are well established and include better compliance, lower cost, and reduction of the potential wash-out effect. ${ }^{6,7}$

Netildex is a fixed steroid/antibiotic combination containing $0.1 \%$ dexamethasone and $0.3 \%$ netilmicin. Dexamethasone is one of the most widely used corticosteroids

Table 4 Effect on clinical secondary parameters (FA dataset)

\begin{tabular}{llll}
\hline Clinical parameters & \multicolumn{2}{l}{ (Score difference) } & P-value* \\
\cline { 2 - 3 } & Netildex & Tobradex & \\
\hline Conjunctival edema & $1.04 \pm 0.96$ & $0.76 \pm 0.82$ & 0.134 \\
Conjunctival discharge & $0.96 \pm 0.58$ & $0.98 \pm 0.30$ & 0.975 \\
Lid hyperemia & $\mathrm{I} .02 \pm 0.89$ & $0.62 \pm 0.62$ & $\mathbf{0 . 0 1 6}$ \\
Lid edema & $0.84 \pm 0.94$ & $0.53 \pm 0.69$ & 0.093 \\
Pain & $0.85 \pm 0.93$ & $0.65 \pm 0.75$ & 0.332 \\
Photophobia & $\mathrm{I} .36 \pm 0.9 \mathrm{I}$ & $\mathrm{I} .16 \pm 0.79$ & 0.339 \\
Tearing & $\mathrm{I} .71 \pm 0.90$ & $\mathrm{I} .18 \pm 0.77$ & $\mathbf{0 . 0 0 I}$ \\
Burning & $\mathrm{I} .5 \mathrm{I} \pm 0.8 \mathrm{I}$ & $\mathrm{I} .13 \pm 0.77$ & $\mathbf{0 . 0 0 7}$ \\
Stinging & $\mathrm{I} .27 \pm 0.76$ & $0.85 \pm 0.80$ & $\mathbf{0 . 0 0 4}$ \\
Foreign body sensation & $\mathrm{I} .29 \pm 0.92$ & $\mathrm{I} .16 \pm 0.60$ & 0.464 \\
\hline
\end{tabular}

Notes: All clinical parameters were evaluated by using a categorical semiquantitative grading scale as described in Materials and methods. The score difference for each parameter at Day 6 from baseline was evaluated. *Wilcoxon rank-sum test. Values in bold are statistically significant.

Abbreviation: FA, full-analysis dataset. 
in ophthalmology and has proven highly effective in treating ocular inflammation. ${ }^{15,16}$ Netilmicin is a third-generation aminoglycoside characterized by a wide spectrum of antimicrobial activity, ${ }^{8-11}$ a low level of conjunctival and corneal toxicity, ${ }^{17,18}$ and a high efficacy rate in treating bacterial conjunctivitis. ${ }^{10,19}$ The combination product of dexamethasone and netilmicin is already available in some EU and non-EU countries in preserved multidose bottles and preservative-free unidoses, and is indicated for steroidresponsive ocular inflammations with or at risk of ocular infection. This combination has already been shown to be safe and effective in controlling ocular inflammation after cataract surgery. ${ }^{12,13}$

In the present study, we compared the clinical efficacy of Netildex with that of Tobradex in 139 patients with signs of conjunctival inflammation and conjunctival discharge requiring an antibiotic treatment. The results of this randomized, double-blind clinical study demonstrated that the two fixed steroid/antibiotic combinations were equivalent in reducing signs and symptoms. Equivalence between Netildex and Tobradex was demonstrated for the primary efficacy parameter (conjunctival hyperemia) at the endpoint visit (Day 6). The magnitude of the reduction in conjunctival hyperemia (approximately 70\%) following treatment was as expected for those treatments, and similar to that previously reported..$^{20,21}$ The responder rate (the percentage of patients demonstrating a clinical significant improvement) was high for both products ( $87 \%$ and $91 \%$ for the Netildex and Tobradex groups, respectively). Patients experienced less burning, stinging, and tearing with Netildex, which may be related to the formulation (solution versus suspension); however, the significance of this finding is not clear, and may not be clinically significant.

Our data confirm that bacterial pathogens are isolated from a low percentage of patients with clinical signs of conjunctivitis (one-third in the present study). This finding has little effect on the clinical effectiveness of a steroid/ antibiotic combination. Nevertheless, a high eradication rate was observed in case of infection in all patients regardless of the drug used, confirming the efficacy of aminoglycosides in treating infections of the anterior segment of the eye.

\section{Conclusion}

The results of this study confirm that Netildex is as effective and safe as Tobradex in reducing signs and symptoms of external ocular inflammation. The presence of netilmicin guarantees a low prevalence of antibiotic resistance ${ }^{8-11}$ and no epithelial toxicity. ${ }^{17,18}$ In addition, due to the water solubility of the two active ingredients (dexamethasone phosphate and netilmicin sulfate), Netildex is available in a ready-to-use, preservative-free solution, rather than a suspension. This formulation allows dose uniformity, does not require shaking before use, and provides high comfort upon instillation.

\section{Acknowledgments}

We thank all the physicians involved in the clinical study (026/SI Study group): P Vaona, F Faraldi, L Palanza, and E Fornara (Torino, Italy); S Azzaro, A Fede, M Parisi, G Stella, and N Fallisi (Ragusa, Italy); G Boccassini, F Rosa, B Kropp, A Balestrazzi, L Rapagnetta, C De Stefano, T Marini Padovan, and M Iossa (Roma, Italy); D Spinelli, MR Curatola, and S Franceschin (Milano, Italy); L Mastropasqua, M Ciancaglini, and G Gambino (Chieti, Italy); L Bauchiero, D Belli, and A Ossella (Ivrea, Italy); and A Nicodin, Dr I Manoliu, and Dr C Iliuta (Bucharest, Romania). We thank also Dr Eileen Collazo for her writing support.

\section{Disclosure}

The authors report no conflict of interest in this work. Vincenzo Papa, Daria Rasà, Debora Santoro, and Simona Russo are, or have been, employees of the manufacturer (SIFI SpA) of the product described in this article. Francesco Faraldi has no commercial or proprietary interest in the product described in this article.

\section{References}

1. Mahmood AR, Narang AT. Diagnosis and management of the acute red eye. Emerg Med Clin North Am. 2008;26(1):35-55.

2. Wirbelauer C. Management of the red eye for the primary care physician. Am J Med. 2006;119(4):302-306.

3. Leibowitz HM. The red eye. N Engl J Med. 2000;343(5):345-351.

4. Cronau H, Kankanala RM, Mauger T. Diagnosis and management of red eye in primary care. Am Fam Physician. 2010;81(2):137-144.

5. Everitt H, Little P. How do GPs diagnose and manage acute infective conjunctivitis? A GP survey. Fam Pract. 2002;19(6):658-660.

6. Leibowitz HM, Pratt MV, Flagstad IJ, Berrospi AR, Kundsin R. Human conjunctivitis II. Treatment. Arch Ophthalmol.1976;94:1752-1756.

7. Barber BL, Strahlman ER, Laibovitz R, Guess HA, Reines SA. Validation of a questionnaire for comparing the tolerability of ophthalmic medications. Ophthalmology. 1997;104(2):334-342.

8. Campoli-Richards DM, Chaplin S, Sayce RH. Netilmicin. A review of its antibacterial activity, pharmacokinetic properties and therapeutic use. Drugs. 1989;38(5):703-756.

9. Bonfiglio G, Scuderi AC, Russo G. Netilmicin: in vitro activity, time-kill evaluation and postantibiotic effect on microorganisms isolated from ocular infections. Chemotherapy. 2001;47(2):117-122.

10. Papa V, Aragona P, Scuderi AC, et al. Treatment of acute bacterial conjunctivitis with topical netilmicin. Cornea. 2002;21(1):43-47.

11. Blanco AR, Sudano Roccaro A, Spoto CG, Papa V. Susceptibility of methicillin-resistant staphylococci clinical isolate sto netilmicin and other antibiotics commonly used in ophthalmic therapy. Curr Eye Res. [Epub March 27, 2013.] 
12. Russo S, Papa V, Di Bella A, et al. Dexamethasone-netilmicin: a new ophthalmic steroid-antibiotic combination. Efficacy and safety after cataract surgery. Eye (Lond). 2007;21(1):58-64.

13. Pianini V, Passani A, Rossi GCM, Passani F. Efficacy and safety of netilmicin/dexamethasone preservative-free and tobramycin/ dexamethasone-preserved fixed combination in patients after cataract surgery. J Ocul Pharmacol Ther. 2010;26(6):617-621.

14. Cagle GD, Abshire RL. Quantitative ocular bacteriology: a method for enumeration and identification of bacteria from the skin-lask margin and conjunctiva. Invest Ophthalmol Vis Sci. 1981;20(6):751-757.

15. Jaanus SD, Cheetham JK, Lesher GA. Anti-inflammatory drugs. In: Bartlett JD, Jaanus SD, editors. Clinical Ocular Pharmacology, 4th ed. Woburn (MA): Butterworth-Heinemann; 2001:265-298.

16. Sherif Z, Pleyer U. Corticosteroids in ophthalmology: past-presentfuture. Ophthalmologica. 2002;216(5):305-315.

17. Papa V, Leonardi A, Getuli C, Pacelli V, Russo P, Milazzo G. Effect of ofloxacin and netilmicin on human corneal and conjunctival cells in vitro. J Ocul Pharmacol Ther. 2003;19(6):535-545.
18. Marino C, Paladino GM, Scuderi AC, Trombetta F, Mugridge K, Enea V. In vivo toxicity of netilmicin and ofloxacin on intact and mechanically damaged eyes of rabbit. Cornea. 2005;24(6):710-716.

19. Milazzo G, Papa V, Carstocea B, et al. Topical netilmicin compared with tobramycin in the treatment of external ocular infection. Int J Clin Pharmacol Ther. 1999;37(5):243-248.

20. White EM, Macy JI, Bateman KM, Comstock TL. Comparison of the safety and efficacy of loteprednol $0.5 \%$ /tobramycin $0.3 \%$ with dexamethasone $0.1 \%$ /tobramycin $0.3 \%$ in the treatment of blepharokeratoconjunctivitis. Curr Med Res Opin. 2008;24(1): 287-296.

21. Rhee SS, Mah FS. Comparison of tobramycin $0.3 \%$ /dexamethasone $0.1 \%$ and tobramycin $0.3 \%$ /loteprednol $0.5 \%$ in the management of blepharo-keratoconjunctivitis. Adv Ther. 2007;24(1):60-67.
Clinical Ophthalmology

\section{Publish your work in this journal}

Clinical Ophthalmology is an international, peer-reviewed journal covering all subspecialties within ophthalmology. Key topics include: Optometry; Visual science; Pharmacology and drug therapy in eye diseases; Basic Sciences; Primary and Secondary eye care; Patient Safety and Quality of Care Improvements. This journal is indexed on

\section{Dovepress}

PubMed Central and CAS, and is the official journal of The Society of Clinical Ophthalmology (SCO). The manuscript management system is completely online and includes a very quick and fair peer-review system, which is all easy to use. Visit http://www.dovepress.com/ testimonials.php to read real quotes from published authors. 